\title{
Sclero-Atrophic Reaction after Botulinum Toxin Injection
}

\author{
Persechino $S^{1 *}$, Lupi $F^{1}$, Di Vito E ${ }^{1}$, Romano ${ }^{1}$, Persechino $F^{2}$, Parisella FR ${ }^{3}$, De Marco $G^{1}$, Tammaro $A^{1}$ \\ ${ }^{1}$ NESMOS Department, Dermatology Unit, Sant'Andrea Hospital, Faculty of Medicine and Psychology, University of Rome "Sapienza" \\ ${ }^{2}$ Dermatology Unit, University of Modena, Italy. \\ ${ }^{3}$ Faculty of Medicine, Towson University, Towson City, Maryland (USA)
}

Received: April 04, 2016; Accepted: April 20, 2016; Published: April 29, 2016

*Corresponding author: Severino Persechino, PhD, NESMOS Department, Dermatology Unit, Sant'Andrea Hospital, Faculty of Medicine and Psychology, Via di Grottarossa, 1035, 00189 Rome, Italy, Phone: +39-06-33775269; Fax: +39-06-33775378; E-mail: severino.persechino@uniroma1.it

\begin{abstract}
We describe a case of sclero-atrophy which is localized to the frontal margin of the scalp's insertion, in a 55 years-old woman, following the local injection of botulinum toxin type $\mathrm{A}$.

After 10 days from the drug application for aesthetic purposes, the patient presented pruritic erythematous reaction and subsequent sclero-atrophic reaction, with hair loss and reduction off ollicularostia. Moreover, were observed telangiectasias and folliculitis, in some points. Skin biopsy showed sclerosis of the dermal-epidermal junction and the reticular dermis, in a context of lymphocytic chronic inflammation with perivascular and periadnexal distribution. Three hairy follicular structures are visible in cross-section, one of which is in complete sclero-atrophy, and the remaining two with initial perifollicular sclerosis.
\end{abstract}

Botulinum toxin is a neurotoxin that inhibits release of acetylcholine at the neuromuscular junction. Injection of small quantities of it into specific overactive muscles causes localized muscle relaxation that smooths the overlying skin and reduces wrinkles.

The case just described could be a sclerosis consequent to the drug injection. Hypotheses may be different, including an inappropriate application of the toxin, probably in a seat higher than the usual one, then going to involve the fibrous tissue of the galea capitis, rather than muscle; or else, an overdose of the drug that causes a super saturation of cholinergic receptors and subsequent amplification of the effect on the surrounding tissues.

The case we present is very striking, since the histological and topographic features associated with the botulinum toxin injection, have not yet been reported in the literature.

Keywords: Botulinum toxin; Sclera-atrophy reaction; Wrinkles; Cholinergic receptors

\section{Introduction}

The "dermal sclero-atrophic reaction" is an alteration of the skin characterized by atrophy and sclerosis, that is affixing of collagen at the level of the dermal-epidermal junction and of the reticular dermis, in the context of which there is a lymphocytic chronic inflammationwith perivascular and periadnexal distribution. Clinically it presents with thickening and hardening of the skin and with reduction of follicular ostia.
The injection of botulinum toxin for the treatment of facial wrinkles is the cosmetological treatment more frequently performed. The most commonly treated sites are the frontal, periorbital and perioral regions [1,3].

Botulinum toxin is a potent neurotoxin that inhibits release of acetylcholine at the neuromuscular junction. Injection of small quantities of it into specific overactive muscles causes localized muscle relaxation, which smoothes the overlying skin and reduces wrinkles. Injection of botulinum toxin is contraindicated in people with keloids, neuromuscular disorders (myasthenia gravis), allergies to botox components, and body's dysmorphic disorder [5].

Adverse effects include bleeding, bruising, swelling, erythema, asymmetry, skin nodules, and pain during injection. Other complications are scarring, hyper / hypopigmentation, infections, damage to the deeper structures, inadvertent intraarterial injection, loss of sight, skin necrosis, granulomas, allergic reactions, product migration, chronic inflammation, lymphoedema, stiffness of tissues. [2,4].

\section{Materials and Methods}

We describe a case of sclero-atrophy which is localized to the frontal margin of the scalp's insertion, in a 55 years-old woman, following the local injection of botulinum toxin type A.

After 10 days from the drug application for aesthetic purposes, the patient presented pruriticerythematous reaction and subsequent sclero-atrophic reaction, with hair loss and reduction off ollicularostia. Moreover, were observed telangiectasias and folliculitis, in some points. The patient, also, showed a net margin (step) between healthy skin and reactive lesion and in some isolated points was observed an active follicular process (Figure 1).

Skin biopsy figure 2 showed sclerosis of the dermal-epidermal junction and the reticular dermis, in a context of lymphocytic chronic inflammation with perivascular and periadnexal distribution. Three hairy follicular structures are visible in crosssection, one of which is in complete sclero-atrophy, and the remaining two with initial perifollicular sclerosis. 

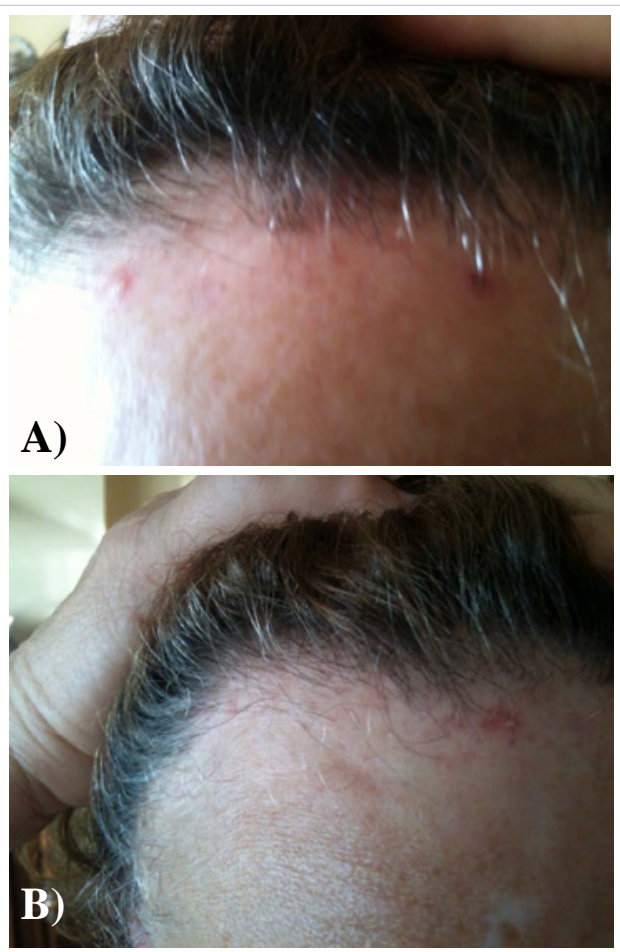

Figure 1: Patient with sclera-atrophy, folliculitis, hair loss and reduction of follicular ostia, after local injection of botulinum toxin type A.

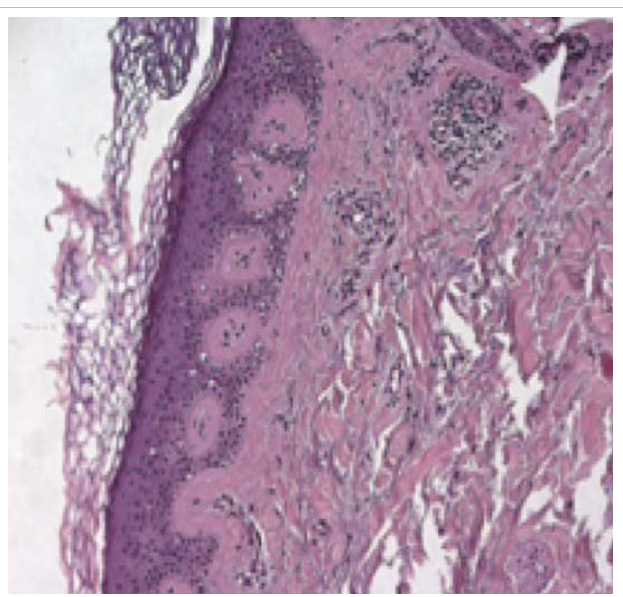

Figure 2: Mild sclerosis of dermo-epidermal junction and of reticular dermis associated with chronic perivascular inflammation. H\&E 25X.

\section{Discussion}

The frontal muscle, common site of botulinum toxin injection, born from the eyebrow skin entwining its fibers with the procerus muscle and the orbicularis oculi muscle. At the top ends on the front part of the aponeurotic galea, dense fibrous layer that covers the entire top of the skull and it continues in the two frontal muscles and posteriorly in the two occipital muscles.
At the front, it is prolonged in a narrow beam that creeps in between the two frontal muscles, and splits, at the level of the coronal suture, in two more wide beams, which laterally enclose these two muscles. This region is innervated from the frontal nerve and from the facial nerve, whose somatic motor fibers are distributed to the furriers muscles of the scalp and facial face. The frontal nerve, which is a terminal branch of the ophthalmic nerve, together with the nose-ciliary and lacrimal nerves, penetrates into the orbital cavity through the upper part of the superior orbital fissure; it runs along the orbital cavity in contact with the periosteum above the levator palpebrae superioris muscle. Along the front course and before the orbital margin, it divides into sovratroclear and supraorbital branches. In particular, the latter is the one that most innervating the skin of the scalp and frontal region. The anatomical region just described is rich in cholinergicmuscarinic receptors located on postganglionic parasympathetic fibers, on which the botulinum toxin acts by blocking the release of acetylcholine, and causing muscle paralysis [5-7].

\section{Conclusions}

The case just described could be a sclerosis consequent to the drug injection. Hypotheses may be different, including an inappropriate application of the toxin, probably in a seat higher than the usual one, then going to involve the fibrous tissue of the galeacapitis, rather than muscle; or else, an overdose of the drug that causes a super saturation of cholinergic receptors and subsequent amplification of the effect on the surrounding tissues.

The case we present is very striking, since the histological and topographic features associated with the botulinum toxin injection, have not yet been reported in the literature.

\section{References}

1. Small R. Botulinum toxin injection for facial wrinkles. Am Fam Physician. 2014;90(3):168-175.

2. Cox SE, Adigun CG, Complications of injectable fillers and neurotoxins. Dermatol Ther. 2011;24(6):524-536. doi: 10.1111/j.15298019.2012.01455.x

3. Lewis C. Botox cosmetic: a look at looking good. FDA Consum. 2002;36(4):11-13.

4. Nestor MS, Ablon GR. Duration of action of abobotulinumtoxina and onabotulinumtoxina: a randomized, double-blind study using a contralateral frontalis model. J Clin Aesthet Dermatol. 2011;4(9):4349.

5. Durand PD, Couto RA, Isakov R, Yoo DB, Azizzadeh B, Guyuron B, et al. Botulinum Toxin and Muscle Atrophy: A Wanted or Unwanted Effect. Aesthet Surg J. 2016;36(4):482-487. doi: 10.1093/asj/sjv208. Epub 2016 Jan 17.

6. Le Louarn C. [Botulinum toxin and facial wrinkles: a new injection procedure]. Ann Chir Plast Esthet. 1998;43(5):526-533. French.

7. Song KH. Botulinum toxin type A injection for the treatment of frown lines. Ann Pharmacother. 1998;32(12):1365-1367. 